\title{
UNDERTAKING MASS MENINGOCOCCAL VACCINATION: CRITERIA AND EXPERIENCES
}

Donald Holt, Krishna Hort

Northern Sydney Area Public Health Unit

Michael Levy, AIDS / Infectious Diseases Branch

NSW Health Department

A outbreak of meningococcal disease in the Northern Sydney Area provided an opportunity for the Area's Public Health Unit (PHU) to review the criteria for undertaking a mass vaccination program and to relate its experiences to other public health professionals. An outbreak was declared in August 1994 after two students attending the same high school developed meningococcal disease within six days of each other. The students were separated by one school year and close contact between them could not be established although the sister of the second case was in the same class as the index case.

Local Accident and Emergency departments and general practitioners (GPs) were alerted but no additional cases were identified. The initial response of the PHU included the distribution of a letter to household and close personal contacts of each of the affected students giving general information about meningococcal disease and advising them to obtain a prescription for an antibiotic (Rifampicin) from their GP. Information about the disease was provided to the school's other students at school meetings and to their parents by letters from the PHU and the school's principal.

After the laboratory report that both meningococcal cases were serogroup $\mathrm{C}$, a meeting of public health, paediatric and infectious disease experts was called by the Area's Director of Public Health to decide what further action might be required. The decision to undertake a mass vaccination program was made using the following criteria:

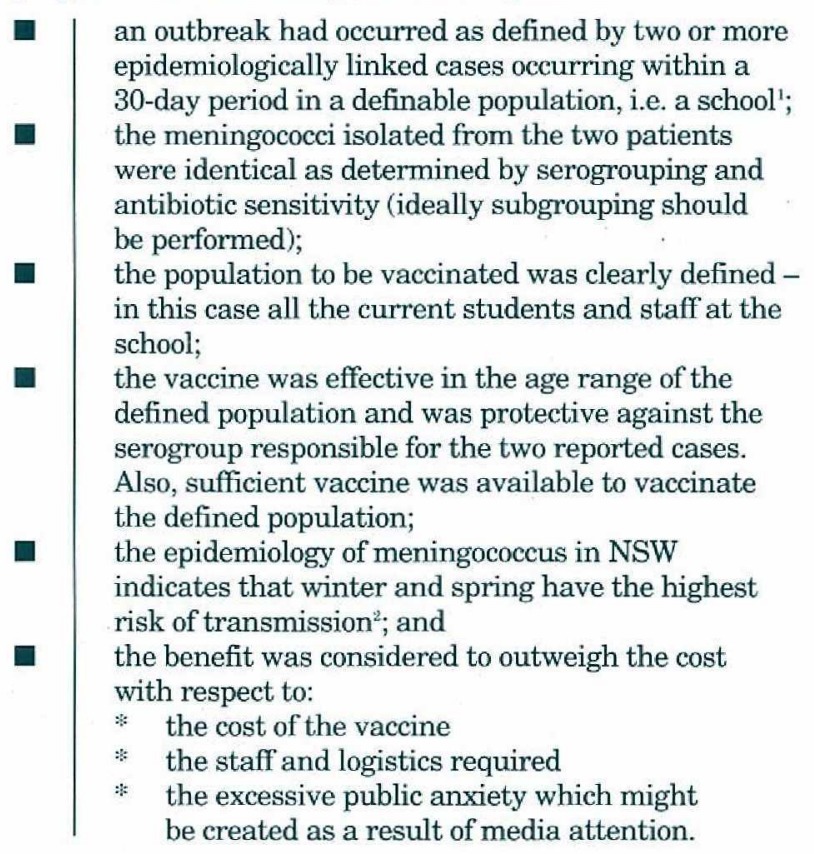

Two vaccination clinics scheduled one week apart were thought necessary because of the numbers to be vaccinated and the problems of trying to get all 700 students to attend one clinic. For example, 5-10 per cent of students are ill and absent from school on any one day.

The logistics required to vaccinate the students and to prepare the necessary letters and media releases occupied
TABLE 3

CRITERIA FOR MASS MENINGOCOCCAL VACCINATION

- Two or more linked cases in a definable population.

- Identical meningococci identified from two (or more) cases.

- The defined population can be vaccinated in a mass program.

- The vaccine is effective:

$i$ against the isolated meningococcal serogroup, and

ii in the defined population.

- The benefit outweighs the cost with respect to:

$i$ the cost of the vaccine

ii manpower and logistics required

iii the excessive public anxiety which might be created due to media attention.

four PHU members full-time for three working days before the first vaccination clinic. Answering telephone inquiries from the public and GPs placed additional demands on the PHU staff over the following week. The school distributed about 700 letters to students which explained to their parents the necessity for a vaccination clinic and enclosed a consent form to be returned before the clinic was to be held. Because only one-third of consent forms had been returned the day before the first clinic, an emergency meeting of all students was called that afternoon. They were advised of the advantages of being vaccinated and offered additional forms if they had lost the first one.

The task of calculating the vaccination uptake rate and identifying students not vaccinated at the first clinic was undertaken by PHU staff who collected consent forms and ticked off vaccinated students on class lists. The time required to mix individual doses of the vaccine with its diluent was the most important factor limiting the rate of vaccination during this clinic. The efficient running of the clinic required sufficient numbers of qualified immunisers; these were arranged by the PHU's public health nurse who drew on hospital, community and council sources.

To increase the uptake at the second clinic, the parents of all students who had not been vaccinated were posted a letter explaining the benefits of vaccination. By the end of the two clinics 590 students (about 85 per cent of the total) and 69 members of staff had been vaccinated.

Allaying public anxiety about meningitis is usually thought to be the most important public health task following an outbreak. By means of telephone contact, letters and meetings, PHU staff worked closely with the school community and GPs to allay anxiety and ensure the successful management of the mass vaccination program. All media inquiries were channelled to the Director of the PHU.

We considered the vaccination program to be successful and at the time of writing this report (six weeks after the index case) no additional cases associated with the outbreak had been notified.

1. Communicable Diseases Standing Committee. A report on the control of meningococcal disease in Australia (third draft). National Health and Medical Research Council, Canberra. April 1994.

2. NSW Health Department. 1992 Infectious Diseases Notifications. NSW Public Health Bulletin 1993; 4(55):25-26. 